\title{
COMMENTARY
}

\section{Assessment of myocardial viability: using coronary pressure and flow after acute myocardial infarction}

\section{J Kern}

Heart 2003;89:9-10

$\Lambda^{\text {s. }}$ sessment of myocardial viability has been a challenge for revascularisation therapeutics in determining the effectiveness of an intervention and patient prognosis. In most cases, the current standard for viability is the recovery of function measured by left ventricular (LV) wall motion and the metabolic activity measured by positron emission tomography (PET) with the uptake of the metabolic tracer 18-fluorodeoxyglucose (FDG). In this issue of Heart, Schimada and colleagues ${ }^{1}$ report how they coupled this imaging methodology of the metabolic activity of viable myocardium with other physiologic indices. In 27 patients, coronary flow and pressure were measured after successful angioplasty in patients undergoing acute myocardial infarction intervention within 12 hours of the onset of symptoms. FDG PET scan results and pressure at zero flow slope index, the SIFP, from simultaneously recorded aortic pressure and coronary flow velocity signals at peak hyperaemia, distal to the lesion in the re-canalised artery were compared. The percentage FDG uptake in the infarct myocardium was normalised for FDG uptake in the non-infarct myocardial region. This ratio was then compared to coronary flow reserve and the SIFP. There was a correlation between the percentage FDG uptake and the SIFP. A significant positive correlation was present, unlike the relation to coronary flow reserve. The authors indicated that the SIFP correlated best with residual myocardial viability and that this index may be an important predictor of viability in patients after acute myocardial infarction.

Of the many tests used to assess myocardial viability, such as PET scanning, thallium reinjection/redistribution imaging, dobutamine stress echocardiography, and coronary flow reserve (hyperaemia in the infarct zone), coronary flow reserve is the weakest. There are no prior studies of the coronary circulation using the SIFP combining simultaneous pressure and flow to couple microvascular tone and epicardial resistance to myocardial viability in the post-infarct setting. The correlation between these variables thus provides new information with regard to the coronary microcirculation and the recovery of myocardial function, both by PET and LV wall motion by echocardiography. It is curious to note that the coronary resistance computations of SIFP but not coronary flow reserve (CFR) correlated with myocardial viability. The finding that an index of coronary resistance is associated with better outcome is in concert with some previous studies and certainly at odds with others, reflecting the diversity of clinical models and potential important contribution of coronary flow as a predictor of viability.

\section{SIFP VERSUS CFR}

Why would SIFP but not CFR correlate with viability? Perhaps the answer lies in the gross measures used in computing CFR, using the ratios of the hyperaemic to basal flow velocity and the fact that this measurement is strongly dependent on both infarct and border zone hyperaemia instead of only true infarct zone flow resistance. In addition, there are many factors which influence CFR, which are not in play for the SIFP relation. The main advantage of the SIFP measurement is to describe the coronary circulatory response and microvascular tone, independent of changes in haemodynamics, or baseline and maximal hypaeremic responses. However, the SIFP relation may be strongly influenced by the quality and mass of the myocardial perfusion bed. Shimada and colleagues ${ }^{1}$ deemed this an unimportant consideration, since there was no correlation between the SIFP and infarct size, but only myocardial viability by PET scanning. These data remain provocative in regard to the establishment of the relation of SIFP for the percentage FDG uptake.

As with all such new observations, this study has limitations, which are well elucidated by the authors. Only 27 subjects were studied; certainly a larger number would help identify any erroneous or outlying data points. The one month FDG PET study may reflect residual myocardial impairment and longer term evaluation may be required to exclude hibernating myocardium and late recovery not associated with an improved SIFP. The use of aortic pressure rather than distal coronary pressure across a diffuse stenosis would enhance the true coronary pressure at zero flow in the post-stenotic region rather than in the proximal artery, assuming no loss of pressure transmission across a diffusely diseased vessel segment or target lesion. Finally, the SIFP may be weakened by a non-linear extrapolation to baseline, which may or may not hold true, even in the acute infarct setting. In fact, the linear extrapolation did not describe the characteristics of the pressure flow relation as plotted during a long diastole, as described by DiMario and colleagues. $^{2}$ Under normal conditions in the cardiac cycle, the pressure at zero flow in general does extrapolate well to a linear point on the baseline hemodynamic axis.

Despite these limitations, the exploration of the pressure at zero flow and the calculation of

Abbreviations: CFR, coronary flow reserve; FDG

18-fluoro-deoxyglucose; LV, left ventricular; PET, positron emission tomography; SIFP, slope index of the

flow-pressure relation 
microvascular resistance in combination with myocardial viability is an advance in our understanding of the coronary circulation. The measurement of pressure at zero flow with a combined pressure and flow sensor guidewire producing data which eliminate the confounding influence of haemodynamics on CFR alone will provide new insights into the effects of reperfusion therapies for acute myocardial infarction.

\section{REFERENCES}

1 Shimada K, Sakanove Y, Kobayashi Y, et al. Assessment of myocardial viability using coronary zero flow pressure after sucessful angioplasty in patients with acute anterior myocardial infarction. Heart 2003;89:71-6. 2 DiMario C, Krams R, Gil R, et al. Slope of the instantaneous hyperemic diastolic coronary flow velocity-pressure relation. Circulation 1994;90:1215-24.

\section{IMAGES IN CARDIOLOGY}

\section{Pituitary apoplexy following anticoagulation for acute coronary syndrome}

A

48 year old man presented with intermittent central chest pain and tingling in the left arm of two days' duration. He had no past medical history of ischaemic heart disease but had been diagnosed with sleep apnoea syndrome 18 months previously and was being treated with continuous positive airway pressure. He was on no medication. General and systemic examination were unremarkable. Resting 12 lead ECG showed sinus rhythm with a normal axis and $1 \mathrm{~mm}$ ST segment depression with $\mathrm{T}$ wave inversion in the inferolateral leads. He was diagnosed as having acute coronary syndrome and treated with aspirin (300 mg dose immediately followed by $75 \mathrm{mg}$ daily), clopidogrel (300 mg dose immediately followed by $75 \mathrm{mg}$ daily), low molecular weight heparin ( $1 \mathrm{mg} / \mathrm{kg}$ body weight twice daily), atenolol $50 \mathrm{mg}$ daily, and nitrate infusion. His ECG reverted to normal and he was pain-free. The troponin I measured 12 and 24 hours after admission were found to be $3.9 \mu \mathrm{g} / \mathrm{l}$ and $4.9 \mu \mathrm{g} / \mathrm{l}$, respectively (normal $<0.1 \mu \mathrm{g} / \mathrm{l}$ ) confirming the diagnosis. Thirty six hours after admission the patient complained of frontal headache and blurring of vision followed by bilateral ptosis with complete right ophthalmoplegia. He had a left third cranial nerve palsy with a partial fourth and a partial sixth cranial nerve palsy. Magnetic resonance imaging scan of the head revealed an extensive pituitary tumour extending into the cavernous sinuses with areas of haemorrhage and infarction (below left and right).

Pituitary function tests at that time revealed high prolactin concentrations of $10072 \mathrm{mu} / \mathrm{l}$, thyroid stimulating hormone $2 \mathrm{mu} / \mathrm{l}$,

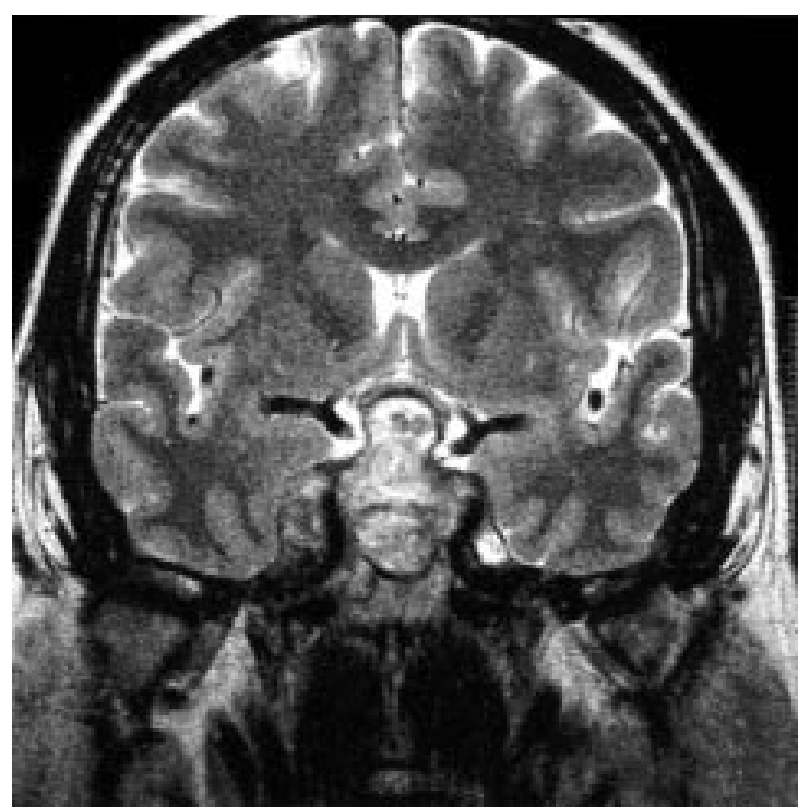

Free $\mathrm{T}_{4} 9.8 \mathrm{pmol} / \mathrm{l}$, cortisol $212 \mathrm{nmol} / \mathrm{l}$, luteinising hormone $1.2 \mathrm{iu} / \mathrm{l}$, follicle stimulating hormone $1.1 \mathrm{iu} / \mathrm{l}$, and growth hormone $1.2 \mathrm{mu} / 1$. Aspirin, low molecular weight heparin, and clopidogrel were discontinued. The patient underwent transsphenoidal decompression of the infarcted pituitary; neurosurgery was uncomplicated. Immunocytochemistry was strongly positive for prolactin. An echocardiogram two weeks following neurosurgery revealed normal left ventricular function confirmed by ventriculogram. Selective coronary angiography showed mild generalised diffuse disease with a moderate/ severe stenosis in the circumflex proximal to the origin of a large obtuse marginal branch. Four weeks following neurosurgery the patient had a 30\% recovery of third and sixth cranial nerve function in the left eye and complete ophthalmoplegia in the right eye.

While the combinations of aspirin/low molecular weight heparin and aspirin/clopidogrel have been shown to be beneficial in acute coronary syndrome, there is no evidence that the use of these three agents in combination have an added beneficial effect without an increase in the risk of major bleeding. The above case serves as a reminder of the possibility of increased bleeding tendency with the use of the above three drugs in combination.

D V Nagarajan

D Bird

M Papouchado Darbhamulla@aol.com

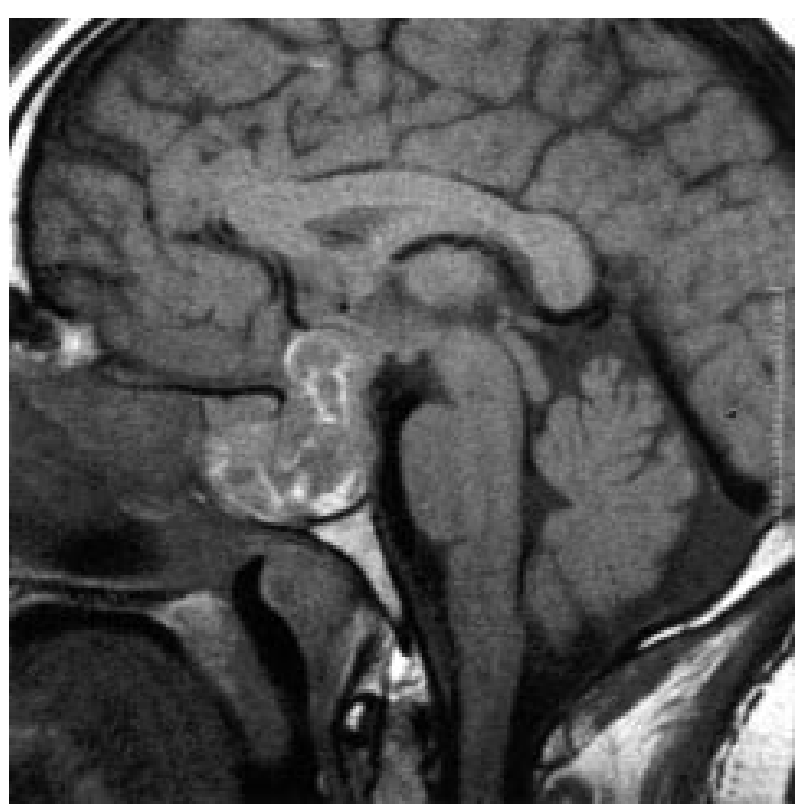

